\title{
Article \\ Applications of Advanced Analysis Technologies in Precise Governance of Social Media Rumors
}

\author{
Xinyu Du ${ }^{1}$, Limei Ou ${ }^{2,3}$, Ye Zhao ${ }^{1}$, Qi Zhang ${ }^{1}$ and Zongmin $\mathrm{Li}^{1}{ }^{1} * \mathbb{D}$ \\ 1 Business School, Sichuan University, Chengdu 610065, China; dxy18583083790@163.com (X.D.); \\ lnykzy_1208@163.com (Y.Z.); 13547802519@163.com (Q.Z.) \\ 2 School of Public Administration, Sichuan University, Chengdu 610065, China; olm@swufe.edu.cn \\ 3 Department of Teaching Affairs, Southwest University of Finance and Economics, Chengdu 611130, China \\ * Correspondence: lizongmin@scu.edu.cn; Tel.: +86-028-8541-5143
}

Citation: Du, X.; Ou, L.; Zhao, Y.; Zhang, Q.; Li, Z. Applications of Advanced Analysis Technologies in Precise Governance of Social Media Rumors. Appl. Sci. 2021, 11, 6726. https://doi.org/10.3390/ app11156726

Academic Editor: Barbara Guidi

Received: 21 May 2021

Accepted: 19 July 2021

Published: 22 July 2021

Publisher's Note: MDPI stays neutral with regard to jurisdictional claims in published maps and institutional affiliations.

Copyright: (c) 2021 by the authors. Licensee MDPI, Basel, Switzerland. This article is an open access article distributed under the terms and conditions of the Creative Commons Attribution (CC BY) license (https:// creativecommons.org/licenses/by/ $4.0 /)$.

\begin{abstract}
Social media rumor precise governance is conducive to better coping with the difficulties of rumor monitoring within massive information and improving rumor governance effectiveness. This paper proposes a conceptual framework of social media rumor precise governance system based on literature mining. Accordingly, insightful directions for achieving social media rumor precise governance are introduced, which includes (1) rational understanding of social media rumors, especially large-scale spreading false rumors and recurring false rumors; (2) clear classification of rumor spreaders/believers/refuters/unbelievers; (3) scientific evaluation of rumor governance effectiveness and capabilities. For the above three directions, advanced analysis technologies applications are then summarized. This paper is beneficial to clarify and promote the promising thought of social media rumor precise governance and create impacts on the technologies' applications in this area.
\end{abstract}

Keywords: rumor governance; social media rumors; literature mining; big data technologies

\section{Introduction}

With the development of the Internet, social media has become a large-scale information dissemination platform and has a huge user base from all sections and age groups of society. The number of users is also rising every year. Millions of people interact on social media and create huge amounts of data. Therefore, vast information provides a living space for rumors. Especially during public health crisis events such as the Covid-19 epidemic, the public's perception is in a state of high uncertainty, which has led to an acceleration of the spread of rumors and aggravated social instability. A rumor can be defined as an assertion that something has happened to some person, group, event or organization, which has not yet been proved true but is transmitted from person to person [1]. Therefore, rumor governance includes verification of true rumors and refutation of false rumors, which plays an important role in calming people's emotions, maintaining social stability, and preventing serious social crises caused by the further deterioration of the incident.

With the awareness of the importance of rumor governance, many online rumor governance platforms have emerged. Their governance method is exactly based on the verification of true rumors and the refutation of false rumors but is more aimed at the second case due to false rumors often cause more serious harm. Taking China as an example, in August 2013, under the leadership of the Beijing Internet Information Office and the Beijing Internet Association, the Joint Rumor Refutation Platform for Websites in Beijing was established (py.qianlong.com/, accessed on 8 February 2021), marking a new era in the construction of rumor governance in China. Nowadays, there are many rumor governance platforms initiated by industry, governments, and Internet companies. According to the "2020 Online Rumor Governance Analysis Report" released by Tencent [2], in 2020, Tencent's "Fact Check" Platform produced 4425 pieces of rumor refutation content, which provided more than 1.72 billion rumor refutation and popular science information. 
These kinds of information and content played a positive role in creating a healthy social media ecosystem.

The characteristics of digitization, network, immediacy, and interaction in the new media era have rapidly affected and changed the mode of rumor generation and dissemination. The rumors also show the characteristics of high quantity, and their spread speed and influence have made great leaps. In the process of rumor governance, it is easy to fall into the ocean of big data without identifying key elements, resulting in inefficient governance. Massive data brings troubles but also opportunities. The precise governance of rumors based on big data and advanced technologies turns traditional extensive management to data-driven precise and scientific governance, which is conducive to the maximum utilization of governance resources and the improvement of governance effectiveness.

At present, there is not much research on the precise governance of social media rumors. This paper is intended to conduct a systematic literature mining of rumor, through which answer following research questions:

(1) How to achieve precise governance of social media rumors?

(2) What specific measures and technologies can achieve precise governance?

The main contributions of this paper can be summarized as:

(1) Reviewing the relevant literature on rumors and clarifying the concept of precise governance of social media rumor.

(2) Summarizing the applications of advanced analysis technologies in social media rumor precise governance and providing insightful ways to achieve precise rumor governance.

This paper is organized as follows. Section 2 presents the literature mining. Section 3 establishes a conceptual framework of social media rumor precise governance. Section 4 summarizes applications of advanced analysis technologies in social media rumor precise governance. Finally, Section 5 provides conclusions.

\section{Literature Mining}

\subsection{Literature Mining of Rumor Research}

To conduct a literature review on rumors, we searched relative literature on Web of Science (WOS) (1959-2021) by using keywords "rumor" and its different spelling "rumour". WOS is the world's largest comprehensive academic database. It includes more than 26,000 world-renowned and high-impact academic journals, and contains various fields of natural sciences, engineering technology, biomedical, and so on. WOS can provide us with relatively comprehensive, formats uniformed and academically quality references and has nice compatibility to CiteSpace and Endnote. The total number of articles we obtained from WOS databases is 2391.

To ensure the accuracy, the following steps were performed.

- Deleting repetitive articles. A total of eight duplicated publications were identified and eliminated by Endnote.

- Adding missing information. Some literature lack publication year information, for they belong to Early Access publications. We manually added publication year to 26 Early Access articles.

- Excluding irrelevant documents. We found that even though the double quotation marks (" ") to perform a precise search, some irrelevant medical literature appeared. We excluded these five irrelevant papers.

In order to summarize the hot spots and trends of rumor research, a systematic literature mining was carried out on the final 2378 documents of Web of Science (WOS) data.

CiteSpace, one of the most popular tools for knowledge mapping, is used for visualizing and analyzing trends and patterns in scientific literature. For the first, we applied this tool to generate a keyword co-occurrence knowledge graph, as shown in Figure 1.

CiteSpace automatically extracts the clustering identification to cluster texts on the relevant literature in the WOS database, and the visual clustering map is shown in Figure 1. The clustering results show that the research hot spots include heterogeneous network, 
social media, financial rumor, rumor detection, and fake news. Public emotion and social ties are also the focuses.

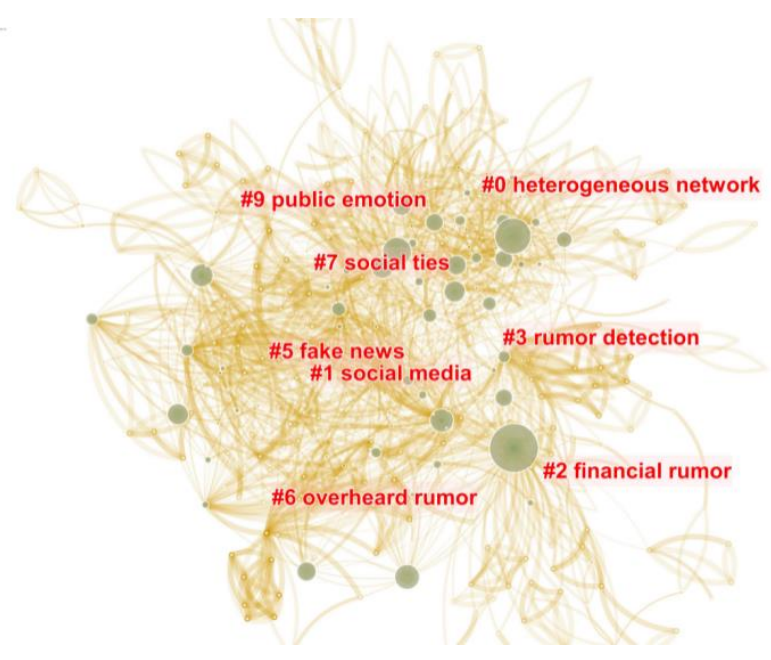

Figure 1. Keyword clustering map of WOS database.

The top 13 keywords with the strongest citation burst in the WOS database are shown in Figure 2. The keywords burst out recently are machine learning, propagation, deep learning, epidemic model, news, and fake news. It can be seen that machine learning is a popular advanced analysis technology recently applied in the research of rumors.

Top 13 Keywords with the Strongest Citation Bursts



Figure 2. Top 13 keywords with the strongest citation bursts in the WOS database.

As it is shown in Figure 3, the distribution of research for different countries from 1959 to 2021 is visualized by Citespace. In a given time period, the thickness of a ring of a circle is proportional to the total number of papers of that country. On the top of the figure is a time bar, and the color of the rings can show different publication times accordingly. If two circles are connected with a line, then there exists co-authorship. Although rumor papers from China were first published as late as 2001, the number of published papers in China ranks second, accounting for $18.33 \%$ of total studies. The top four countries (USA, China, England, France) contributed nearly $50 \%$ of rumor research and dominated this research field in the perspective of the number of publications.

Figure 4 shows a dual-map overlay of the final document storage. The dual-map overlay is designed by Chen and Leydesdorff [3] to reveal patterns of a scientific portfolio with respect to a global map of scientific literature. The visualization of dual-map overlay refers to the relationship between citing and cited. Citespace visualizes the degree of 
concentration of these papers and how these papers connect different regions based on their citation relationships. Each area is represented by a color, standing for a research field. The color curves represent the reference paths, which start from the citing map on the left and point to the cited map on the right. A series of journals that belong to one area can determine the nature of the area, and therefore a cluster can be formed. The most frequently appeared words in the corresponding journals are used to label each area. As it can be seen in Figure 4, papers on rumors primarily appear in three broad areas on the citing map: the area in the top center in red with the label of mathematics/systems/mathematical; the area in the bottom in dark blue with the label of economics/economic/political; and the area near the bottom in light blue with the label of psychology/education/health. The citation curves all point to the area in the cited map on the right, and the most important red, dark blue and light blue links (they are also among the thickest links) are marked with citing and cited journals. Take the citation path in red for an example. This path depicts that publications in mathematics-, systems-, and mathematical- related journals cite primarily one group of journals, including systems/computing/computer.

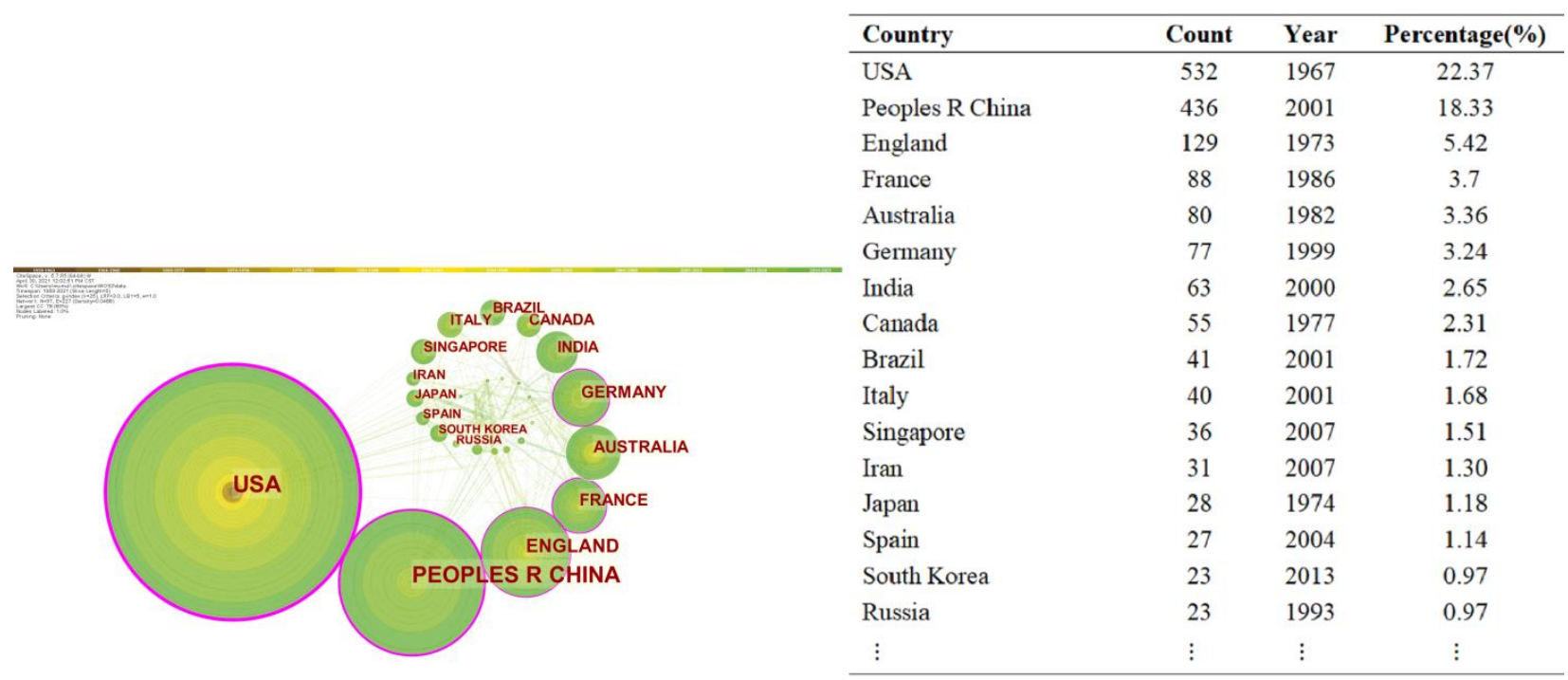

Figure 3. Rumors research distribution in different countries.

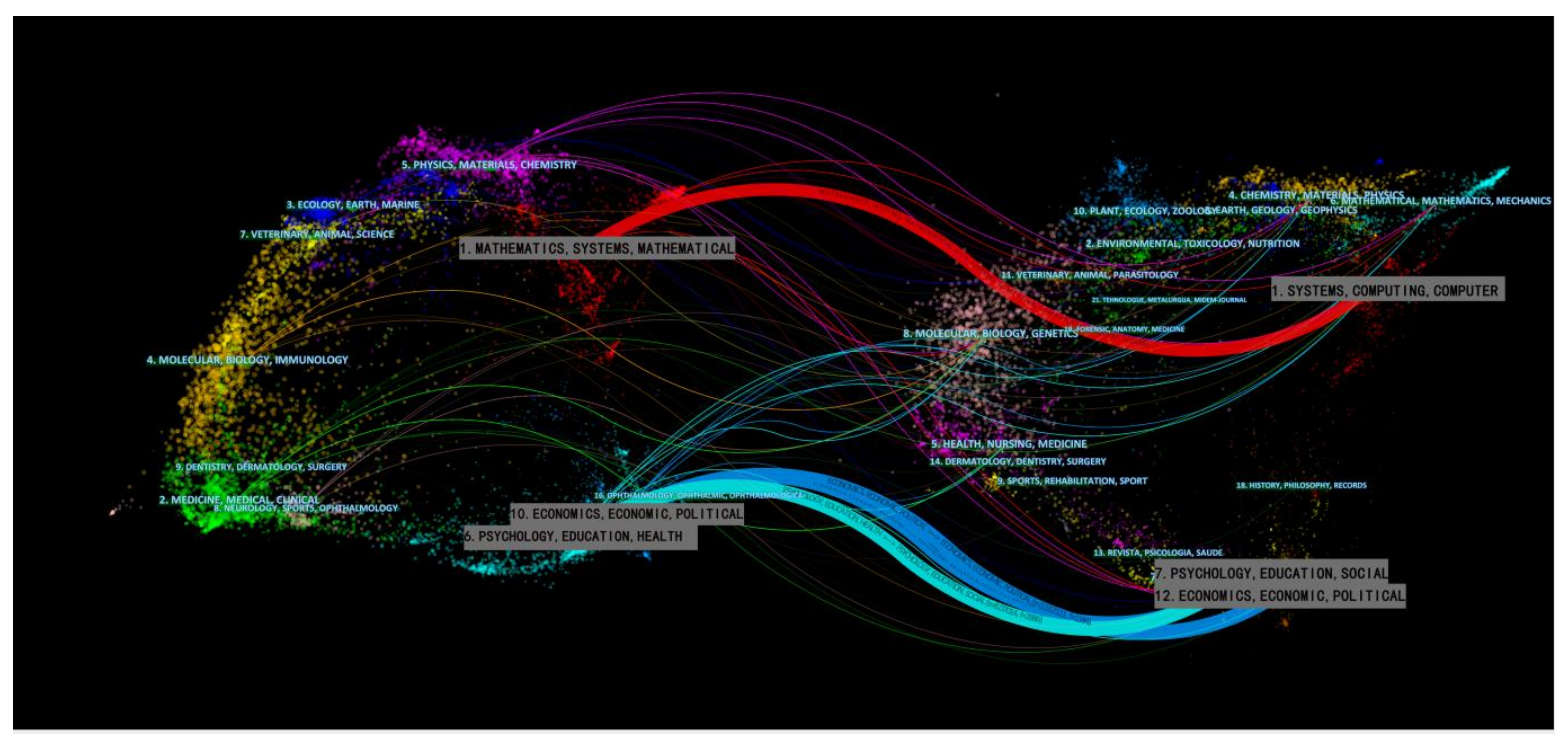

Figure 4. A dual-map overlay of the final document storage. (The color curves represent the reference paths; the most frequently appeared words in the corresponding journals are used to label each area.). 
Figure 5 describes the appearance time of keywords. Each node represents a keyword. The repetition times of keywords in the obtained data are represented by 'tree rings'. The color of the ring indicates when it appears. The thickness of a ring is proportional to the number of appearance frequency in a given time slice. Links between different keywords mean that they appear in the same article at the same time. It can be concluded that rumor spreading, social media, social network, complex network, rumor propagation have received long-term and close attention in the research of rumors. Although the dark red nodes, including machine learning and deep learning, are not prominent in scale, they represent the latest hot technologies in rumors.

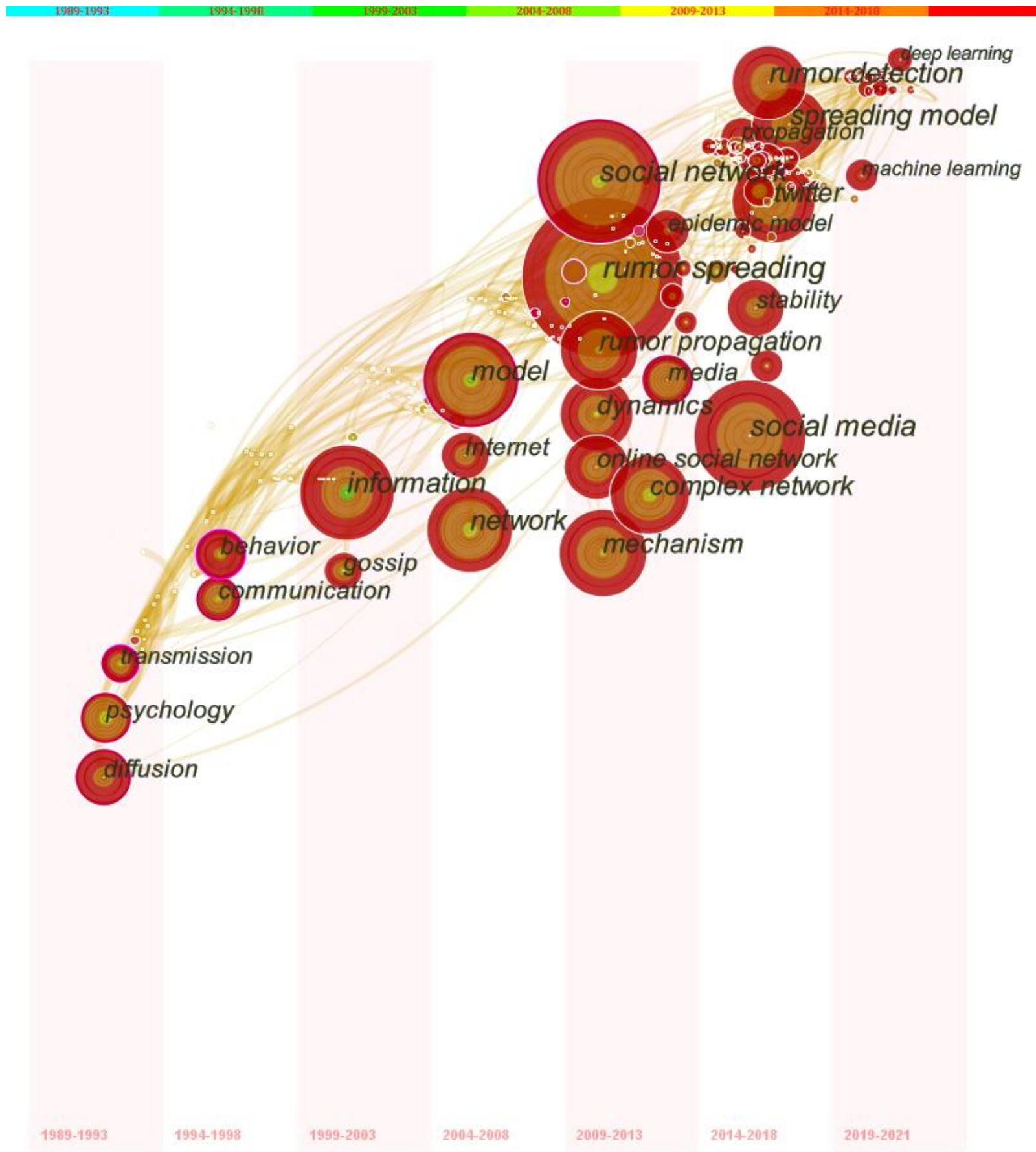

Figure 5. Appearance time of keywords. (The color of a ring denotes the time of appearance; the thickness of a ring is proportional to the number of appearance frequency in a given time slice; the link between different keywords denotes co-occurrence in the same paper.). 


\subsection{Research Categories of Rumors}

The academic research on rumors began with Knapp's research on rumor governance in Massachusetts in 1944 [4]. In recent years, rumors and rumor refutation have gradually become popular research fields. These researches span sociology, psychology, media, and machine learning [5].

A total 2378 documents in the final database are classified into seven categories, as shown in Table 1. Categories of literature are based on sorting out reading literature. We have some initial categories based on previous review papers [6,7]. Then, in the order of citation numbers, we sort out reading literature based on title, keywords and abstract to classify each literature into a proper category. A new category is determined when literature cannot be classified into existing categories. This process continues until no new category is found.

Table 1 sorts out the related literature on rumors and classifies the research into seven categories.

Table 1. Research of rumor classification and technologies.

\begin{tabular}{|c|c|c|c|}
\hline Number & Categories & $\begin{array}{l}\text { Research Directions, Technologies, } \\
\text { and Representative Literature }\end{array}$ & Research Object \\
\hline 1 & Rumor influence & $\begin{array}{l}\text { 1.1. Rumor's impact prediction [8] } \\
\text { 1.2. Rumor influence maximization [9] } \\
\text { 1.3. The extent of rumor influence [10] } \\
\text { 1.4. Analysis of rumor influence [11] }\end{array}$ & \\
\hline 2 & $\begin{array}{l}\text { Rumor diffusion/ } \\
\text { propagation model }\end{array}$ & $\begin{array}{l}\text { 2.1. Stochastic rumor spreading model [12] } \\
\text { 2.2. The rumors tracking [13] } \\
\text { 2.3. D-K Model [14], M-T Model [15] } \\
\text { 2.4. Extensions and improvements of classic SIR Model [16,17] } \\
\text { 2.5. Dynamic models, simulation methods [18] } \\
\text { 2.6. Propagation model based on bio-mathematics theory [19] } \\
\text { 2.7. Research on rumor spreading based on complex network } \\
\text { and social network theory [20-22] }\end{array}$ & Rumor \\
\hline 3 & $\begin{array}{l}\text { Identification or } \\
\text { detection of rumors }\end{array}$ & $\begin{array}{l}\text { 3.1. Rumor identification based on text and topic }[23,24] \\
\text { 3.2. Rumor identification based on propagation structure }[25,26] \\
\text { 3.3. Rumor detection over varying time windows. [27] }\end{array}$ & \\
\hline 4 & $\begin{array}{l}\text { Rumor prevention/ } \\
\text { governance }\end{array}$ & $\begin{array}{l}\text { 4.1. Cross-platform verification [28] } \\
\text { 4.2. Comparison of different rumor governance strategies } \\
\text { [29-31] } \\
\text { 4.3. Rumor governance of emergencies/mass incidents [32] } \\
\text { 4.4. Coordinated strategies across different areas or disciplines } \\
\text { to combat misinformation }[33,34]\end{array}$ & $\begin{array}{l}\text { Platforms and } \\
\text { agencies }\end{array}$ \\
\hline 5 & $\begin{array}{l}\text { Factors of rumor } \\
\text { spreading }\end{array}$ & $\begin{array}{l}\text { 5.1. The influence of self-media on the rumor spreading [35] } \\
\text { 5.2. The influence of attitudes, beliefs/consensus, uncertainty, } \\
\text { skepticism, criticism, polarization, etc., on the rumor } \\
\text { spreading [36-38] } \\
\text { 5.3. The influence of group arguments and opposing views on } \\
\text { the rumor spreading [39,40] } \\
\text { 5.4. The influence of rumor audience's forgetting and hesitation } \\
\text { mechanism on the rumor spreading [41-43] }\end{array}$ & Public \\
\hline
\end{tabular}


Table 1. Cont.

\begin{tabular}{|c|c|c|c|}
\hline Number & Categories & $\begin{array}{l}\text { Research Directions, Technologies, } \\
\text { and Representative Literature }\end{array}$ & Research Object \\
\hline 6 & $\begin{array}{l}\text { The generation of } \\
\text { rumors }\end{array}$ & $\begin{array}{l}\text { 6.1. The psychology of rumors [4] } \\
\text { 6.2. Motivation and basic law of rumors }[44,45]\end{array}$ & \\
\hline 7 & $\begin{array}{l}\text { The social psychology } \\
\text { of rumor spreading }\end{array}$ & $\begin{array}{l}\text { 7.1. Tension theory of cognitive dissonance [46] } \\
\text { 7.2. The theory based on collective memory and behavior [47] }\end{array}$ & \\
\hline
\end{tabular}

Rumor prevention/governance has become a popular rumor research area in recent years. In this area, scholars mainly focus on: (1) case study [29]; (2) from the perspective of psychology, analyzing the effects of different rumor governance methods on eliminating the trust of false rumors and blocking rapid spread [29-31]; (3) from the perspective of different discipline area putting forward some directional suggestions on the spread of rumors $[33,34]$; (4) using the system dynamic model method to study the popularity of rumors, and then proposing governance countermeasures [48]; (5) the social media rumor control models [49], which applies the trust chain/network of social media users to refute false rumors to achieve controlling rumors.

Social media rumors are essentially a kind of public opinion [50], so the precise governance of public opinion is directly related to the precise governance of social media rumors. Some scholars have claimed that precise governance of public opinion can start from the following points [51,52]: (1) establish public opinion prediction and early warning models to achieve accurate judgment and prevention; (2) fine research and judgment of public opinion situation are oriented to realize data-driven decision-making, i.e., artificial intelligence technologies such as data mining and pattern recognition are used to improve research and judgment accuracy of public opinion situation by analyzing the spread and the tendency; (3) the personalized and differentiated public opinion leading assisted by artificial intelligence algorithms makes public opinion leading more effective.

At present, a large number of scholars have discussed the establishment of public opinion prediction and early warning models $[53,54]$, public opinion evolution or tendency [55-57], sentiment analysis, etc. [58,59]. These views and methods have a positive reference for the precise governance of social media rumors.

\subsection{Summary of Literature Mining}

Through the summary of hot spots and trends of rumor research, it can be found that it is a trend to use advanced technologies such as machine learning to conduct research on rumors.

In the current research, big data and advanced analysis technologies [23-26,60-62] are widely used in the recognition and detection of rumors, such as the machine learning methods in recent years. For example, $\mathrm{CNN}$ technology was originally used for image recognition and classification, but now it can be extended for rumor recognition and classification. RNN (recurrent neural network) has good performance in natural language processing (NLP) and time series prediction, so it can be used for rumor recognition and prediction. RF regression can be applied to select the key evaluation indexes.

Rumor literature about rumors conducts theoretical and technical research from three research objects to provide support for rumor governance in general (as shown in Table 1):

Rumor: Most papers study the rumors themselves (Research 1.1-3.3). The rumor diffusion model provides technical support for the characterization of the spread of rumors, and at the same time, it can determine which parameters in the model have significant effects on the spreading process. The detection of rumors also provides technical support for the governance of rumors. 
Platform and agencies: Research 4.1-4.4 focus on existing rumor governance strategies of platforms and institutions or proposing feasible governance recommendations, which have positive support to rumor governance.

Public: A part of the literature focuses on the public in the spread of rumors (Research 5.1-7.2). These papers study the role and influence of the public's psychology and behavior on the generation and dissemination of rumors, and also illustrate the influence of rumors on the public's psychology and behavior.

Through systematic analysis of the rumor research literature, it can be seen that there is still development space in rumor governance. In terms of rumor governance and rumor control decision support, the big data generated by social media rumors in the new media era has not been really used. How to make good use of social media rumor big data and related analysis technologies to provide decision-making support for achieving rumor precise governance and improving the effectiveness of rumor governance also deserves more specific and in-depth exploration.

(1) Few studies have integrated natural sciences and social sciences. Rumor governance is a multidisciplinary research field of psychology, sociology, communication, and management. After a long period of research, in terms of social sciences, qualitative research on dissemination of rumors and social psychology of diffusion has been profoundly accumulated. Although the research in natural sciences started late, in recent years, the research of rumor identification and rumor spreading simulation has progressed rapidly. At present, research in natural sciences and social sciences is relatively independent. In fact, taking the social psychology, mood, emotion, personality, attitude, and other characteristics of the rumor participants into account in rumor governance will help to classify the public so as to design a targeted anti-rumor strategy to break the dilemma of receiving refutation of false rumor. NLP provides technical feasibility, and there is a lot can be done in this respect.

(2) A number of studies have focused on the rumors themselves-the identification, spreading, and influencing factors of rumors, but humans are the main factor in the viral spread of rumors [63]. At the public level, there is no mature research on the group identification of rumors participants and public classification. This aspect plays a key role in improving the precise governance of rumors.

(3) There are now different responding strategies for rumors, and the experimental and case studies on the influence of different strategies on beliefs of rumors have been conducted [29,31]. However, there is still a lack of scientific indicators, systems, and methods for the evaluation of rumor governance effectiveness and capabilities.

In the context of big data, rumor precise governance provides ideas for breaking through the bottleneck of rumor identification in massive information, the dilemma of public's information reception, and the management of rumor refutation platforms' effectiveness, with significant operability. Besides, it also plays an important and positive role in promoting the transformation of social governance from empirical governance and extensive governance to data-driven governance and refined governance.

Therefore, in the context of big data, rumor prediction, group identification, and governance effects and capability evaluation all urgently need further development to provide more targeted decision support for realizing rumor precise governance.

\section{A Conceptual Framework of Social Media Rumor Precise Governance}

Based on literature mining in Section 2, this part presents a conceptual framework of social media rumor precise governance system.

Through literature mining, we conclude that current literature about rumors conducts theoretical and technical research from three research objects to provide support for rumor governance, which are rumor, platforms and agencies, and public. At the same time, how to make good use of social media rumors big data and related analysis technology to provide decision support for realizing accurate rumor governance and improving rumor governance effectiveness is also worthy of in-depth discussion. Therefore, we present a 
conceptual framework of social media rumor precise governance system based on the three research objects construct.

\subsection{From the Perspective of Rumor}

Rumor can be divided into different categories according to its content, such as social rumors (content involving social events and false alarms, etc.), political rumors (content involving policies and political figures, etc.). Regardless of the type, false rumors have negative effects on individuals, organizations, and society. It is recommended to strengthen rational understanding of false rumors and grasp the law and trend of the recurrence and large-scale spread. In the era of new media, the rumor spreading process is greatly accelerated which can lead to an explosion of information in a short period of time and a concentrated outbreak. In comparison, refutation activities are carried out in response to the false rumors which have already been generated. Even with the highest efficiency, the rumor refutation is lagging behind the generation and dissemination of false rumors. At the same time, it should be noted that large-scale false rumors cause greater harm and deeper impact due to the wide coverage. Under limited conditions, false rumors spreading on a large scale should be monitored and tracked. Besides, timely prevention and control should be carried out to reduce the harm. Therefore, we can seize the opportunities and realize accurate rumor governance only if using big data and advanced analysis technologies to dig out the contextual and textual features of large-scale and recurring rumors and carry out effective monitoring and early warning.

\subsection{From the Perspective of Platforms and Agencies}

Internet platforms and agencies are the main participants in the process of rumor governance, mostly perform refutation for false rumors. False rumor refutation platforms mainly include official platforms of national and local governments as well as large platforms that rely on Internet companies, such as China Internet Joint Rumor Refutation Platform, Security Alliance Rumor Refutation Platform, Sina Rumor Refutation Platform, etc. Media organizations and some science popularization organizations are the backbone for rumor refutation, such as the "Truth-Seeking" column on www.People.cn (accessed on 16 April 2021), Ding Xiang Doctor, Tadpole Stave, etc.

For rumor governance platforms/institutions, it is necessary to conduct a scientific evaluation of the effectiveness and capabilities of rumor governance and grasp the key factors to improve the rumor governance. However, there is still a lack of indexes, evaluation systems, and multi-attribute evaluation methods that specifically target the effectiveness of refutation and rumor governance capabilities. If the key factors that affect the effectiveness and ability of rumor governance can be identified, such as the text characteristics, emotional characteristics, and auxiliary pictures/videos of the refutation information, it will help to make targeted improvements. The evaluation results of governance participants can distinguish the advantages and disadvantages of different platforms in terms of resources, credibility for different groups, science popularization capabilities, and technical capabilities. It helps to design a multi-platform coordination refutation response mechanism and refutation paths combination in order to maximize the function of rumor governance participants and improve the effectiveness of refutation.

\subsection{From the Perspective of Public}

The public who participated in the process of rumor spreading and refuting can be divided into the following: (a) rumor spreaders-people who believe in false rumors and spread them; (b) rumor believers- people who believe in false rumors (but not spread); (c) rumor unbelievers - people who do not believe in false rumors; (d) refuters-people who do not believe in false rumors and take the initiative to clarify the rumors. In the process of rumor spreading, platforms and agencies which react to false rumor exert an influence on the public. Further, at the same time, the rumor is also constantly influencing the public's perception. 
It is necessary to distinguish the information expression preferences of different groups of people and different media. The research published by Vosoughi et al. in Science [63] showed that humans themselves were the main factor in the viral spreading of rumorspeople tend to read tweets that are in line with their emotions and attitudes, and think that information that is consistent with their beliefs is more convincing. Therefore, in the face of the existing reception dilemma of refutation information, crowd identification and feature analysis should be realized through big data analysis for different types of false rumors. The main purpose of crowd classification is to push information more accurately based on the characteristics of different groups. For example, to push the refutation information about false commercial rumors to people interested in commercial areas. For users who like to watch videos, platforms can push refutation information with videos; for users who like to read articles, platforms can push refutation information with links to detailed articles. In this way, targeted refutation methods can be designed for potential rumor spreaders/refuters/believers/unbelievers, which is conducive to expanding the acceptance of true news and suppressing the spread of false rumors and is also significant to realizing rumor precise governance.

Through analyzing the three-way support for the precise governance of social media rumors, we propose a conceptual framework as shown in Figure 6.

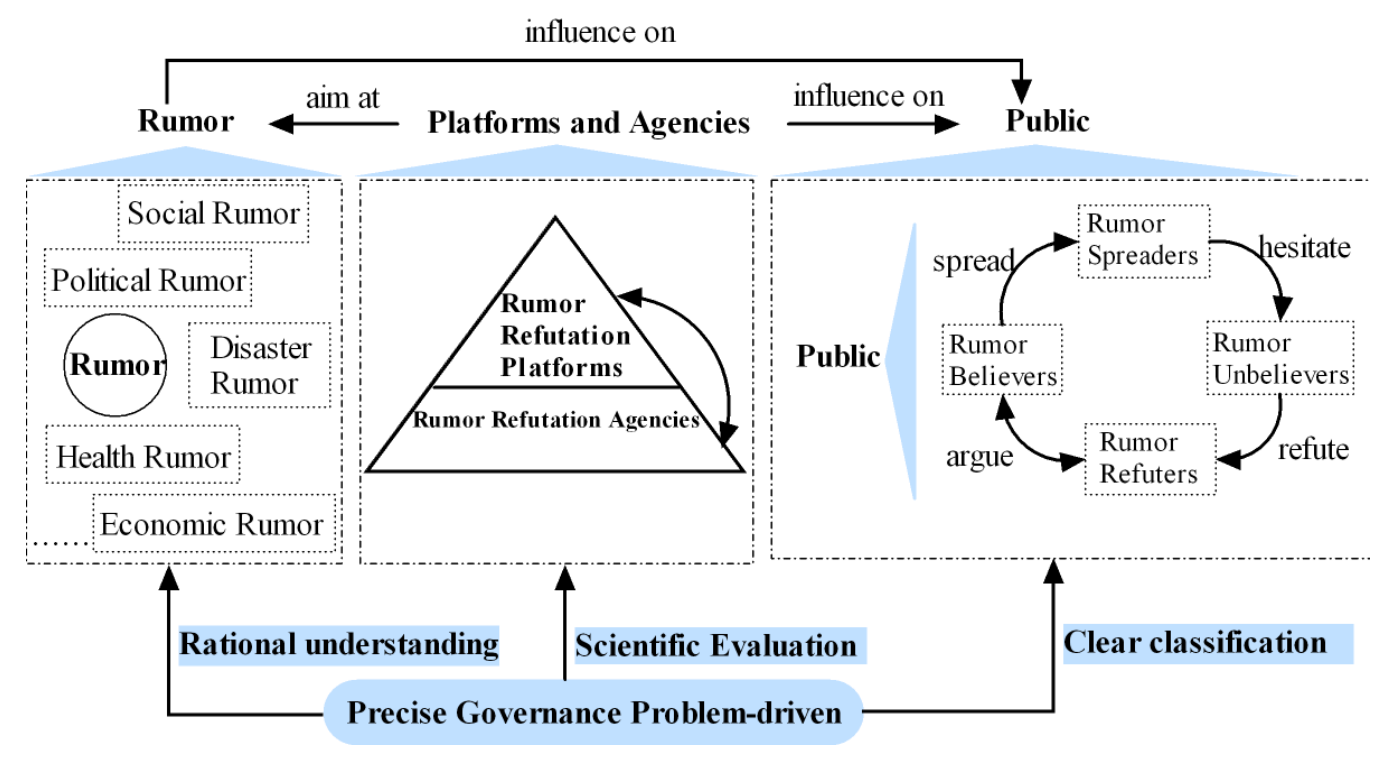

Figure 6. The Framework of Social Media Rumor Precise Governance System.

It can be seen that the rational understanding of rumor, the clear classification of the public, and the scientific evaluation of rumor governance platforms and agencies are viable directions for the precise governance of social media rumors. We can achieve precise rumor governance only if we (1) achieve a rational understanding of rumors in the governance process, grasp the modes and characteristics of various types of false rumors dissemination, and focus on large-scale spreading false rumors and recurring false rumors; (2) clearly classify the public, extract the characteristics of various groups of people such as rumor spreaders/believers/refuters/unbelievers and govern according to different groups; (3) achieve a scientific evaluation of governance participants and identification of key factors that improve governance effectiveness and capabilities.

\section{Applications of Advanced Analysis Technologies in Social Media Rumor Precise Governance}

The development of artificial intelligence and machine learning has made it possible to crawl large amounts of data and process and extract unstructured data. Three different ways can provide the decision-making basis for the precise governance of social media 
rumors: establishing a large database of social media rumors and rumor spreading participants and using advanced big data analysis to extract the characteristics of large-scale spreading and recurring false rumors; accurately identifying and predicting the rumor believers, rumor spreaders, rumor unbelievers and rumor refuters of social media rumors; exploring the factors which have significant impacts on the effectiveness/ability of rumor governance.

\subsection{Applications of Advanced Analysis Technologies in Identification and Monitoring of Large-Scale Spreading and Recurring Social Media False Rumors}

The Internet produces false information, unconfirmed information, and erroneous information all the time. If the rumor governance does not grasp the emphasis, it is easy to fall into the "ocean" of information. The focus of governance is the large-scale and recurring false rumors.

Whether rumors spread on a large scale was originally a binary classification problem, in order to increase the pertinence of preventive decision-making, a multi-class classification problem of high risk, medium risk, and low risk can be defined in accordance with the spreading risk. Researchers can establish a large database of false social media and use machine learning methods to predict and classify rumors with different spread risk levels. Besides, feature engineering can also be used to screen the characteristics of large-scale spreading false rumors. The characteristics extracted from different types of possible large-scale spreading false rumors provide a basis for the refutation platforms to formulate preventive countermeasures. Knowing the current situational state (variability, danger, sensitivity, local informatization level, etc.), information verified as false rumors appear in the monitoring system of the rumor refutation platforms. Analyzing its originators and text characteristics and taking these characteristics into the predictive model can quickly predict whether the false rumors will spread on a large scale or the risk level of large-scale spreading. In this way, we can take pertinent preventive measures based on the predicted results and establish a hierarchical early warning system. According to the rumor refutation experience, different levels of emergency refutation plans formed from the aspect of the governance participants, phases, measures, etc., to improve the pertinence and effectiveness of refutation.

The recurring false rumors are divided into the following categories according to the repetition cycle: (1) intergenerational rumors-spread across generations, with a rotation cycle of several years; (2) annual ring rumors-recurring during specific regular social activities and time; (3) "menstrual" rumors-do not target specific events, but appear at intervals of irregular time throughout the long period; (4) "influenza" rumors-similar rumors reappear when similar emergencies (natural disasters, accidents, etc.) occur. It is advisable to explore recurring rumors' characteristics and identify possible recurring rumors based on the rumor database and advanced big data technologies. That is to say, establishing machine learning prediction models and figuring out whether the refutation method, organization, refutation speed, and platforms during rumor's first appearance have a significant impact on the probability of rumor recurring. In order to reduce the possibility of rumor recurring to a certain target level, it is worth exploring what methods, organization, speed, and platforms need to be adopted to refute rumors so as to provide a decision-making basis for preventing the false rumor from recurring.

In recent years, the advanced technology applied in the detection of social media rumors is also abundant. For example, Nasir et al. [24] proposed a model based on advanced technology and proved the efficiency of deep learning models in detecting fake news. In the work of Wang et al. [60], a novel two-layer cascaded gated recurrent unit (CGRU) model based on the SD and the TsDTS algorithm was proposed for rumor events detection, named as SD-TsDTS-CGRU, which outperformed the latest rumor events detection algorithms. Kotteti et al. [61] proposed an ensemble model, and performed a majority-voting scheme on the prediction set of neural networks, and used the time series vector representation of Twitter data to quickly detect rumors. Tu et al. [62] proposed a novel rumor detection framework named Rumor2vec, consisting of joint text and propagation structure represen- 
tation learning. Experiments based on early rumor detection showed that their method could recognize rumor at least $12 \mathrm{~h}$ ahead of other methods.

\subsection{Applications of Advanced Analysis Technologies in Crowd Identification and Classification}

Lewandowsky et al. believed that the same refutation information should be rewritten in different statements and perspectives according to different people's characteristics and thinking patterns, avoiding sensitive points such as political stand and world view [64]. This requires crowd classification of the public, that is, the target of rumor governance. In addition, the group prediction model can also be used to predict potential false rumor spreaders. From this perspective, it is possible to distinguish the opinions and information expression preferences of different groups of people and to adopt rumor refutation decisions for different groups. This can break through the dilemma of receiving refutation information and realize the effective dissemination of refutation information. For more details:

Based on the group classification model, the participants of rumor governance can push the popular science knowledge or policy popularization information to the past rumors participants according to the crowd classification results. For example, for the former false rumor spreaders, push the corresponding type of science information and remind them to pay attention to content review when publishing information. Secondly, for those who did not believe in false rumors but have not yet become active rumor refuters, push platform incentive policies information, such as rumor refutation rewards points. Besides, for false rumor refuters, the platform can also push incentive policies information and other types of refutation information that they are interested in, encouraging them to continue to be active science popularizers and purifiers on the Internet. If their reposts cause a certain influence, the platform should honor the incentives in time, such as gift points, virtual gifts, membership level upgrades. In this way, a false rumor refutation platform can establish a long-term incentive mechanism that can advocate folk rumor refutation, reward science popularization, and promote continuous emergence of science popularization talents. Lastly, for rumor believers, the platform should regularly push the same type of science popularization information to carry out science popularization education for them to avoid being confused by similar false rumors.

From the follows and tags of the rumor participants, platforms can mine the preferences of different groups for different media and try to push targeted information through the media that they are interested in and trust. In addition, the platform can push refutation information of false rumors corresponding to the interest preferences of different groups.

According to the group prediction model, when a false rumor appears or is in the process of spreading, platforms can predict who among the public may become rumor refuters, rumor spreaders, rumor believers, and unbelievers. Then relevant popular science information can be early pushed, trying to make science popularization or rumor refutation repeatedly before the rumor information reaches them to achieve "rumor immunity".

Furthermore, for participants of different types of rumors, personality analysis can be conducted from the information and comments that they have proactively released recently. These participants can be further classified in detail so that the pushed information can be revised to suit their personalities.

Combination of multiple information forms. Information on social media networks is composed of various forms, mainly including text, pictures, video, voice, etc. The multiple expression forms increase the expressive power of the information. The dissemination of refutation information should make full use of the group's preference for the information types and design the most acceptable information forms for the group so that the masses will eventually accept the refutation content and science information content. This helps to form the correct information perspective to improve the acceptance of information.

There are many advanced technologies applied in group identification and classification. Indu et al. [65] have developed a novel algorithm based on the Forest Fire model. The model used the identified important features to obtain the probability of a node to spread a 
rumor. By detecting the rumor-impacted nodes in the network, the extent of rumor spread across a network was measured. The proposed method can also depict the propagation of rumors, and the key nodes which played a vital role in spreading the rumor can also be identified. Li et al. [66] applied NLP techniques to quantify the user's sentiment tendency and recent interests. Then, those results were combined with other personalized features to train an XGBoost classification model, and potential rumor refuters can be identified from various rumor events in aspects of economics, society, disaster, politics, and military. Furthermore, agreement and disagreement views analysis marked with attributes can also be applied in crowd classification by NLP. In addition, some machine learning algorithms, such as LR, RF, SVM, GBDT, XGBoost, are potential tools in group identification and classification. GBDT is one of the best algorithms that fit the true distribution in traditional machine learning algorithms, and it can be used for classification and feature selection. XGBoost is widely used in various fields due to its high accuracy, parallel processing, and portability. In future research, the above algorithms can be applied to the crowd identification and classification of social rumor refutation.

\subsection{Applications of Advanced Analysis Technologies in Rumor Governance Effectiveness/Capabilities Evaluation}

Although people have made efforts to control rumors, there are still widespread rumors, even repeated, intensified with deeper impact. In addition to the rapid development of the Internet and other objective factors, to a large extent, it is due to the lack of refutation effectiveness / capabilities evaluation and failure to grasp key factors to improve the effect of false rumor refutation.

Li et al. [67] proposed a rumor refutation effectiveness index (denoted as REI) to evaluate the effectiveness of a rumor refutation microblog on social media. NLP approaches and other big data analysis tools make it convenient to identify the emotional polarity of the comments, extract the characteristics of the text and measure the sentiment of a rumor refutation microblog. To explore the relationship between REI and its possible affecting factors, conventional linear regression model and three regression models from the machine learning field, i.e., support vector regression model (SVR), extreme gradient boosting regression model (XGBoostRegressor), and light gradient boosting machine regression model (LGBMRegressor), are compared. Based on the model with the best performance, they provided useful suggestions on enhancing the rumor refutation effectiveness.

Many advanced technologies, such as multi-attribute decision making, group decision making, fuzzy comprehensive evaluation, have been applied in the evaluation problems in different areas. Not much attention has been paid to false rumor refutation effectiveness/capabilities evaluation, and it can be a potential research direction in the future. Feasible and practical suggestions can be proposed for false rumor refutation participants when we can evaluate their effectiveness/capabilities properly. At the same time, combining the different advantages and disadvantages of different platforms, we can propose a multi-platform collaborative refutation response mechanism and a combination of refutation paths, including:

(1) The topics of online rumors cover a wide range of fields and involve different public groups. It is supposed to adopt a differentiated multi-subjects combination strategy to gradually integrate the official false rumor refutation force and the private refutation force with the understanding of the advantages and disadvantages of various rumor governance participants. Finally, different platforms will be complementary of each other to concentrate advantages and make up for disadvantages. Therefore, a rapid, targeted, and precise governance mechanism will be formed.

(2) The integrates of government force and multimedia channels. Newspapers and news are mainly official reports and one-way communication. False rumor refutation platforms initiated by national, industry, local governments, and Internet companies form an interactive communication form depend on the open comment. WeChat Moments have a closed-loop communication effect, and WeChat public accounts and Apps form the tribe of people who have the same interest. With the help of the authority of official 
reports and the democracy of open platforms, the omni-media convergence and omnichannels disseminating for refutation information can be realized. According to the group's characteristics and media preference, we can design the combination of refutation paths for different types of false rumors.

\section{Conclusions}

This paper studied applications of advanced analysis technologies in social media rumor precise governance through literature mining and the establishment of a precise governance conceptual framework for the social media rumors. From systematic literature mining, we found that advanced technology is very popular in the current rumor research. Current research mainly focuses on three objects: the rumor, platforms and agencies, and the public. Therefore, we construct the framework based on these three research objects. Accordingly, insightful directions for achieving social media rumor precise governance are introduced, which includes (1) rational understanding of social media rumors, especially large-scale spreading false rumors and recurring false rumors; (2) clear classification of rumor spreaders/believers/refuters/unbelievers; and (3) scientific evaluation of rumor governance effectiveness and capabilities. Finally, according to the framework, some advanced analysis technologies applied in rumor precise governance are summarized.

From the perspective of advanced analysis technologies, this paper sheds light on all aspects of social media rumor precise governance system: rumors, platforms and agencies, and public. This paper is beneficial to clarify and promote the promising thought of social media rumor precise governance and create impacts on the technologies' applications in this area.

Author Contributions: Conceptualization, Z.L.; writing, X.D., Y.Z., L.O.; original draft, X.D., Y.Z.; validation, L.O., Q.Z.; funding acquisition, Z.L. All authors have read and agreed to the published version of the manuscript.

Funding: This research was supported by the Major Project of National Social Science Foundation of China (Grant No. 17ZDA286), National Natural Science Foundation of China (Grant No. 71640013), and the Fundamental Research Funds for the Central Universities (Grant No. SXYPY202117).

Institutional Review Board Statement: Not applicable.

Informed Consent Statement: Not applicable.

Data Availability Statement: This statement can be excluded.

Conflicts of Interest: The authors declare no conflict of interest.

\section{References}

1. Sunstein, C. On Rumors: How Falsehoods Spread, Why We Believe Them. Straus and Giroux: What Can Be Done; Farrar: New York, NY, USA, 2009.

2. Financial Headlines. The 2020 Sunshine Media Workers Conference Was Held, Leading Content Entrepreneurs to a Positive Ecological View. Available online: https: / cj.sina.com.cn/articles/view/1704103183/65928d0f0200253ts (accessed on 8 February 2021). (In Chinese).

3. Chen, C.; Leydesdorff, L. Patterns of connections and movements in dual-Map overlays: A new method of publication portfolio analysis. J. Assoc. Inf. Sci. Technol. 2014, 65, 334-351. [CrossRef]

4. Knapp, H.R. Apsychology of Rumor. Public Opin. Q. 1944, 8, 22-37. [CrossRef]

5. Ahsan, M.; Kumari, M.; Sharma, T.P. Rumors detection, verification and controlling mechanisms in online social networks: A survey. Online Soc. Netw. Media 2019, 14, 100050. [CrossRef]

6. Pal, A.; Chua, A.Y.K. Analysis of Research on Online Rumors. In Proceedings of the 2019 5th International Conference on Information Management (ICIM), Cambridge, UK, 24-27 March 2019; pp. 108-112.

7. Li, M.; Wang, X.; Gao, K.; Zhang, S. A survey on information diffusion in online social networks: Models and methods. Information 2017, 8, 118. [CrossRef]

8. Yin, J.; Liu, S.; Li, Q.; Xu, G. Prediction and Analysis of Rumour's Impact on Social Media. In Proceedings of the 2019 6th International Conference on Behavioral, Economic and Socio-Cultural Computing (BESC), Beijing, China, 28-30 October 2020.

9. Vega-Oliveros, D.A.; Costa, L.D.F.; Rodrigues, F.A. Influence maximization by rumor spreading on correlated networks through community identification. Commun. Nonlinear Sci. Numer. Simul. 2020, 83, 105094. [CrossRef] 
10. Grinberg, N.; Joseph, K.; Friedland, L.; Swire-Thompson, B.; Lazer, D. Fake news on Twitter during the 2016 US presidential election. Science 2019, 363, 374-378. [CrossRef]

11. Kwak, N.; Lane, D.S.; Zhu, Q.F.; Lee, S.S.; Weeks, B.E. Political Rumor Communication on Instant Messaging Platforms: Relationships with Political Participation and Knowledge. Int. J. Commun. 2020, 14, 5663-5685.

12. Jain, A.; Dhar, J.; Gupt, V. Stochastic model of rumor propagation dynamics on homogeneous social network with expert interaction and fluctuations in contact transmissions. Phys. A Stat. Mech. Appl. 2019, 519, 227-236. [CrossRef]

13. Li, G.; Dong, M.; Ming, L.; Luo, C.; Yu, H.; Hu, X.; Zheng, B. Deep reinforcement learning based ensemble model for rumor tracking. Inf. Syst. 2021, 101772. [CrossRef]

14. Daley, D.J.; Kendal, D.G. Epidemics and rumors. Nature 1964, 204, 204. [CrossRef]

15. Maki, D.; Thomson, M. Mathematical Models and Applications; Prentice-Hall: Englewood Cliff, NJ, USA, 1973.

16. Yang, A.; Huang, X.; Cai, X.; Zhu, X.; Lu, L. ILSR rumor spreading model with degree in complex network. Phys. A Stat. Mech. Appl. 2019, 531, 121807. [CrossRef]

17. Mathur, A.; Gupta, C.P. Dynamic SEIZ in Online Social Networks: Epidemiological Modeling of Untrue Information. Int. J. Adv. Comput. Sci. Appl. 2020, 11, 577-585.

18. Zhu, L.; Zhou, M.; Liu, Y.; Zhang, Z. Nonlinear dynamic analysis and optimum control of reaction-diffusion rumor propagation models in both homogeneous and heterogeneous networks. J. Math. Anal. Appl. 2021, 502, 125260. [CrossRef]

19. Zhang, Y.; Xu, J. A Dynamic Competition and Predation Model for Rumor and Rumor-Refutation. IEEE Access 2021, 9, 9117-9129. [CrossRef]

20. Ai, S.; Hong, S.; Zheng, X.; Wang, Y.; Liu, X. CSRT rumor spreading model based on complex network. Int. J. Intell. Syst. 2021. [CrossRef]

21. Li, J.; Jiang, H.; Yu, Z.; Hu, C. Dynamical analysis of rumor spreading model in homogeneous complex networks. Appl. Math. Comput. 2019, 359, 374-385. [CrossRef]

22. Zhu, L.; Yang, F.; Guan, G.; Zhang, Z. Modeling the dynamics of rumor diffusion over complex networks. Inf. Sci. 2021, 562, 240-258. [CrossRef]

23. Xu, F.; Sheng, V.S.; Wang, M. Near real-time topic-driven rumor detection in source microblogs. Knowl. Based Syst. 2020, 207, 106391. [CrossRef]

24. Nasir, J.A.; Khan, O.S.; Varlamis, I. Fake news detection: A hybrid CNN-RNN based deep learning approach. Int. J. Inf. Manag. Data Insights 2021, 1, 100007.

25. Huang, Q.; Zhou, C.; Wu, J.; Liu, L.; Wang, B. Deep spatial-temporal structure learning for rumor detection on Twitter. Neural Comput. Appl. 2020, 3, 1-11. [CrossRef]

26. Yu, K.; Jiang, H.; Li, T.; Han, S.; Wu, X. Data Fusion Oriented Graph Convolution Network Model for Rumor Detection. IEEE Trans. Netw. Serv. Manag. 2020, 17, 2171-2181. [CrossRef]

27. Kwon, S.; Cha, M.; Jung, K. Rumor Detection over Varying Time Windows. PLoS ONE 2017, 12, e0168344. [CrossRef] [PubMed]

28. Wen, W.; Su, S.; Yu, Z. Cross-Lingual Cross-Platform Rumor Verification Pivoting on Multimedia Content. Empirical Methods in Natural Language Processing. In Proceedings of the 2018 Conference on Empirical Methods in Natural Language Processing, Brussels, Belgium, 31 October-4 November 2018.

29. Paek, H.; Hove, T. Effective strategies for responding to rumors about risks: The case of radiation-contaminated food in South Korea. Public Relat. Rev. 2019, 45, 101762. [CrossRef] [PubMed]

30. Ozturk, P.; Li, H.; Sakamoto, Y. Combating rumor spread on social media: The effectiveness of refutation and warning. In Proceedings of the 2015 48th Hawaii International Conference on System Sciences, Kauai, HI, USA, 5-8 January 2015; pp. 2406-2414.

31. Clayton, K.; Blair, S.; Busam, J.A.; Forstner, S.; Glance, J.; Green, G.; Kawata, A.; Kovvuri, A.; Martin, J.; Morgan, E.; et al. Real Solutions for Fake News? Measuring the Effectiveness of General Warnings and Fact-Check Tags in Reducing Belief in False Stories on Social Media. Political Behav. 2020, 42, 1073-1095.

32. Lian, Y.; Liu, Y.J.; Dong, X.F. Strategies for controlling false online information during natural disasters: The case of Typhoon Mangkhut in China. Technol. Soc. 2020, 62, 10. [CrossRef]

33. Trethewey, S.P. Strategies to combat medical misinformation on social media. BMJ 2020, 96, 4-6. [CrossRef]

34. Farrell, J.; McConnell, K.; Brulle, R. Evidence-based strategies to combat scientific misinformation. Nat. Clim. Chang. 2019, 9, 191-195. [CrossRef]

35. Li, J.; Song, B.; Luo, C.; Zhang, B.; Wang, S.; Wu, G. Considering Self-media Influence Network Rumor Propagation Model and Control Strategy. In Proceedings of the 2020 IEEE 4th Information Technology, Networking, Electronic and Automation Control Conference (ITNEC), Chongqing, China, 12-14 June 2020.

36. Hu, Y.; Pan, Q.; Hou, W.; He, M. Rumor spreading model with the different attitudes towards rumors. Phys. A Stat. Mech. Appl. 2018, 502, 331-344. [CrossRef]

37. Lu, P. Heterogeneity, judgment, and social trust of agents in rumor spreading. Appl. Math. Comput. 2019, 350, 447-461. [CrossRef]

38. Del Vicario, M.; Bessi, A.; Zollo, F.; Petroni, F.; Scala, A.; Caldarelli, G.; Stanley, H.E.; Quattrociocchi, W. The spreading of misinformation online. Proc. Natl. Acad. Sci. USA 2016, 113, 554-559. [CrossRef] [PubMed]

39. Wang, Q.; Yang, X.; Xi, W. Effects of group arguments on rumor belief and transmission in online communities: An information cascade and group polarization perspective. Inf. Manag. 2018, 55, 441-449. [CrossRef] 
40. Bodaghi, A.; Goliaei, S. A Novel Model for Rumor Spreading on Social Networks with Considering the Influence of Dissenting Opinions. Adv. Complex Syst. 2018, 21, 1850011. [CrossRef]

41. Xu, H.; Li, T.; Liu, X.; Liu, W.; Dong, J. Spreading dynamics of an online social rumor model with psychological factors on scale-free networks. Phys. A Stat. Mech. Appl. 2019, 525, 234-246. [CrossRef]

42. Dong, X.; Liu, Y.; Wu, C.; Lian, Y.; Tang, D. A double-identity rumor spreading model. Phys. A Stat. Mech. Appl. 2019, 528, 121479. [CrossRef]

43. Qiu, X.; Zhao, L.; Wang, J.; Wang, X.; Wang, Q. Effects of time-dependent diffusion behaviors on the rumor spreading in social networks. Phys. Lett. A 2016, 380, 2054-2063. [CrossRef]

44. Rosnow, R.L. On rumor. J. Commun. 1974, 24, 26-38. [CrossRef]

45. Chorus, A. The basic law of rumor. J. Abnorm. Soc. Psychol. 1952, 48, 313-314. [CrossRef]

46. Aronson, E. The Social Animal; Worth Publishers: New York, NY, USA, 1999.

47. Popenoe, D. Sociology; Prentice Hall: Englewood Cliffs, NJ, USA, 1995.

48. Myilsamy, K.; Kumar, M.S.; Kumar, A.S. Optimal control of a rumor model with group propagation over complex networks. Int J. Mod. Phys. C 2021, 32, 27. [CrossRef]

49. Tripathy, R.M.; Bagchi, A.; Mehta, S. Towards combating rumors in social networks: Models and metrics. Intell. Data Anal. 2013, 17, 149-175. [CrossRef]

50. Liang, J.; Yang, M. On spreading and controlling of online rumors in we-media era. Asian Cult. Hist. 2015, 7, 42. [CrossRef]

51. Ren, Y.; Li, Z. Internet Public Opinion Management in Big Data Era. In Proceedings of the 2018 5th International Conference on Education, Management, Arts, Economics and Social Science (ICEMAESS 2018), Sanya, China, 10 November-11 November 2018; pp. $1165-1169$.

52. Mei, Y.; Tu, Y.; Xie, K.; Ye, Y.; Shen, W. Internet Public Opinion Risk Grading under Emergency Event Based on AHPSort II-DEMATEL. Sustainability 2019, 11, 4440. [CrossRef]

53. Peng, L.J.; Shao, X.G.; Huang, W.M. Research on the Early-Warning Model of Network Public Opinion of Major Emergencies. IEEE Access 2021, 9, 44162-44172.

54. Skoric, M.M.; Liu, J.; Jaidka, K. Electoral and Public Opinion Forecasts with Social Media Data: A Meta-Analysis. Information 2020, 11, 187. [CrossRef]

55. Cotfas, L.A.; Delcea, C.; Roxin, I.; Ioanăş, C.; Gherai, D.S.; Tajariol, F. The Longest Month: Analyzing COVID-19 Vaccination Opinions Dynamics from Tweets in the Month Following the First Vaccine Announcement. IEEE Access 2021, 9, 33203-33223. [CrossRef]

56. D'Andrea, E.; Ducange, P.; Bechini, A.; Renda, A.; Marcelloni, F. Monitoring the public opinion about the vaccination topic from tweets analysis. Expert Syst. Appl. 2019, 116, 209-226. [CrossRef]

57. Ma, X.; Liu, W.; Zhou, X.; Qin, C.; Chen, Y.; Xiang, Y.; Zhang, X.; Zhao, M. Evolution of online public opinion during meteorological disasters. Environ. Hazards 2020, 19, 375-397. [CrossRef]

58. Chakraborty, K.; Bhatia, S.; Bhattacharyya, S.; Platos, J.; Bag, R.; Hassanien, A.E. Sentiment Analysis of COVID-19 tweets by Deep Learning Classifiers-A study to show how popularity is affecting accuracy in social media. Appl. Soft Comput. 2020, 97, 14. [CrossRef] [PubMed]

59. Aldiansyah, M.R.; Sasongko, P.S. Twitter Sentiment Analysis About Public Opinion on 4G Smartfren Network Services Using Convolutional Neural Network. In Proceedings of the 2019 3rd International Conference on Informatics and Computational Sciences (ICICoS), Semarang, Indonesia, 29-30 October 2019; pp. 1-6.

60. Wang, Z.; Guo, Y. Rumor Events Detection Enhanced by Encoding Sentimental Information into Time Series Division and Word Representations. Neurocomputing 2020, 397, 224-243. [CrossRef]

61. Kotteti, C.; Dong, X.; Qian, L. Ensemble Deep Learning on Time-Series Representation of Tweets for Rumor Detection in Social Media. Appl. Sci. 2020, 10, 7541. [CrossRef]

62. Tu, K.; Chen, C.; Hou, C.; Yuan, J.; Li, J.; Yuan, X. Rumor2vec: A Rumor Detection Framework with Joint Text and Propagation Structure Representation Learning. Inf. Sci. 2021, 560, 137-151. [CrossRef]

63. Vosoughi, S.; Deb, R.; Sinan, A. The spread of true and false news online. Science 2018, 359, 1146-1151. [CrossRef] [PubMed]

64. Lewandowsky, S.; Ecker, U.K.; Seifert, C.M.; Schwarz, N.; Cook, J. Misinformation and Its Correction: Continued Influence and Successful Debiasing. Psychol. Sci. Public Interest 2012, 13, 106-131. [CrossRef]

65. Indu, V.; Thampi, S.M. A nature-Inspired approach based on Forest Fire model for modeling rumor propagation in social networks-Science Direct. J. Netw. Comput. Appl. 2019, 125, 28-41. [CrossRef]

66. Li, Z.; Zhang, Q.; Wang, Y.; Wang, S. Social Media Rumor Refuter Feature Analysis and Crowd Identification Based on XGBoost and NLP. Appl. Sci. 2020, 10, 4711. [CrossRef]

67. Li, Z.; Zhang, Q.; Du, X.; Ma, Y.; Wang, S. Social media rumor refutation effectiveness: Evaluation, modelling and enhancement. Inf. Process. Manag. 2021, 58, 102420. [CrossRef] 